\title{
The complete genome sequence of CrRV-Ch01, a new member of the family Rhabdoviridae in the parasitic copepod Caligus rogercresseyi present on farmed Atlantic salmon (Salmo salar) in Chile
}

\author{
Arnfinn Lodden Økland ${ }^{1} \cdot$ Renate Hvidsten Skoge ${ }^{1} \cdot$ Are Nylund $^{1}$
}

Received: 6 November 2017 / Accepted: 1 February 2018 / Published online: 14 February 2018

(c) The Author(s) 2018. This article is an open access publication

\begin{abstract}
We have determined the complete genome sequence of a new rhabdovirus, tentatively named Caligus rogercresseyi rhabdovirus Ch01 (CrRV-Ch01), which was found in the parasite Caligus rogercresseyi, present on farmed Atlantic salmon (Salmo salar) in Chile. The genome encodes the five canonical rhabdovirus proteins in addition to an unknown protein, in the order N-P-M-U (unknown)-G-L. Phylogenetic analysis showed that the virus clusters with two rhabdoviruses (Lepeophtheirus salmonis rhabdovirus No9 and Lepeophtheirus salmonis rhabdovirus No127) obtained from another parasitic caligid, Lepeophtheirus salmonis, present on farmed Atlantic salmon on the west coast of Norway.
\end{abstract}

The parasitic copepod C. rogercresseyi (sea louse) is a serious problem for farming of Atlantic salmon in Chile [1-3]. There are no published studies of viruses infecting C. rogercresseyi, but sequences of possible rhabdovirus origin ( $\mathrm{N}$ protein, $\mathrm{G}$ protein and RNA-dependent RNA polymerase genes) have been detected in the parasite. However, these sequences have been suggested to be possible endogenous viral elements (EVEs), i.e., viruses integrated in the germline genome of their hosts and inherited vertically [4-14], or integrated rhabdoviral elements (IREs), i.e., "fossil" traces of extinct rhabdoviruses, and not exogenous rhabdoviruses [8]. This was also suggested to be the case for rhabdovirus-like sequences found in genomes of $L$. salmonis [8], but Økland et al. [15] showed that these EVEs were actual pieces from two different rhabdoviruses infecting this parasite. Hence, the focus of this study was to identify an exogenous rhabdovirus in C. rogercresseyi using EVEs/

Handling Editor: Chan-Shing Lin.

Electronic supplementary material The online version of this article (https://doi.org/10.1007/s00705-018-3768-z) contains supplementary material, which is available to authorized users.

Arnfinn Lodden Økland

arnfinn.okland@uib.no

1 Department of Biology, University of Bergen, 5020 Bergen, Norway
IREs available in the GenBank database (NCBI) as a starting point.

Sequences resembling partial rhabdovirus genomes were identified by BLAST search [16] using several rhabdoviruses as query sequence and limiting the search to $C$. rogercresseyi (taxid: 217165). Additional sequences where identified using BioEdit [17] to perform a local BLAST search in a database constructed from a $C$. rogercresseyi transcriptome shotgun assembly (PRJNA234316). A rhabdovirus genome sequence $(11,529$ nucleotides) based on three sequences (GenBank accession numbers BT075815, GAZX01041482 and GAZX01041484) was assembled using Contig Express from the Vector NTI ${ }^{\circledR}$ Suite 9.0.0 (Thermo Fisher).

The complete genome sequence (11,599 nucleotides) of the new rhabdovirus, named CrRV-Ch01 (accession no: KY203909), was obtained by Sanger sequencing of RNA from $C$. rogercresseyi, using the assembled genome sequence as the basis for primer design. The lice were collected from the skin of farmed Atlantic salmon at three farming sites in Los Lagos (Region X) on the coast of Chile in 2016. The three farming sites are located with an average distance of $65 \mathrm{~km}$. The parasites were fixed in RNAlater ${ }^{\circledR}$ (Thermo Fisher) and transported to the University of Bergen, Norway. RNA was extracted from the lice using TRI Reagent (Sigma-Aldrich), with the following modifications of the standard protocol: The lice were homogenized in 1 $\mathrm{ml}$ TRI Reagent with a $5-\mathrm{mm}$ bead for $7 \mathrm{~min}$ at $50 \mathrm{~Hz}$ in a Tissuelyser LT (QIAGEN), and the RNA pellet was washed with an additional $1 \mathrm{ml}$ of $100 \%$ ethanol before air-drying. 
RNA from 4-5 pooled lice from one location was ligated to allow circularization and sequencing of the genome termini of CrRV-Ch01. To increase the efficiency of RNA ligation, the 5'-triphosphate residues of the RNA were removed by incubating $5 \mu \mathrm{g}$ of total RNA with 5 units of 5 ' RNA pyrophosphohydrolase (Rpph; New England Biolabs) in 40 $\mu 1$ of $1 \mathrm{X}$ NEBuffer 2 for $30 \mathrm{~min}$ at $37{ }^{\circ} \mathrm{C}$ [18], followed by RNA clean-up using an RNeasy Mini Kit (QIAGEN). Four hundred ng of purified dephosphorylated RNA was then ligated for $1 \mathrm{~h}$ at $37{ }^{\circ} \mathrm{C}$ using $10 \mathrm{U}$ of T4 RNA ligase (Thermo Scientific) in $20-\mu 1$ of $1 \mathrm{x}$ reaction buffer for T4 RNA ligase supplemented with $0.1 \mathrm{mg}$ of BSA per $\mathrm{ml}$ and 40 units of RNaseOUT TM (Invitrogen). For cDNA synthesis, $2.5 \mu$ l of ligated RNA was used directly as template for SuperScript ${ }^{\mathrm{TM}}$ III reverse transcriptase (SuperScript ${ }^{\mathrm{TM}}$ III First-Strand Synthesis System for RT-PCR, Invitrogen), with gene-specific primers annealing to the $\mathrm{L}$ gene in the genomic RNA. The cDNA was subjected to nested PCR with forward primers located within the 3' end of the L gene and reverse primers located within the 5' end of the $\mathrm{N}$ gene (Table 1), using the Expand ${ }^{\mathrm{TM}}$ High Fidelity PCR System (Roche). Finally, the nested PCR products were gel purified (QIAquick ${ }^{\circledR}$ Gel Extraction Kit, QIAGEN) and sequenced by the Sanger method.

The resulting complete virus genome sequence of CrRVCh01 differed by $1.1 \%$ from the assembled sequence in the GenBank database and contained six open reading frames (ORFs) that were at least 408 nucleotides (136 amino acids) in length, in the order 3'-N-P-M-U-G-L-5', i.e., different from the standard five-gene arrangement found in many of the rhabdoviruses. The leader region of CrRV-Ch01 is 78 nucleotides long, while the trailer region is 67 nucleotides long. The first 21 nucleotides of the leader region and the last 21 nucleotides of the trailer region exhibit an inverse complementarity of $81.8 \%$. The putative transcription termination/polyadenylation signal (TTP), based on its homology to other rhabdoviruses, is conserved in the genome of CrRVCh01 and comprises the motif TATG(A)7. The transcription initiation (TI) sequence, AACAA, is present for all genes except for the U protein gene, which has AATAA as its TI sequence.

The putative nucleoprotein gene ( $\mathrm{N}$ gene) in $\mathrm{CrRV}-\mathrm{Ch} 01$ is $1425 \mathrm{nt}$ long and contains a single ORF of $1356 \mathrm{nt}$ encoding a putative protein of 452 amino acids (accession number: APF32073). The putative N protein of CrRV-Ch01 shows 98.3\% nucleotide sequence identity $(98.0 \%$ amino acid sequence similarity) to a partial putative rhabdovirus nucleoprotein sequence (accession no: FK898446, 953 nucleotides) obtained from $C$. rogercresseyi in the Pacific Ocean (Chile). Further amino acid sequence comparisons with other rhabdoviruses using BLAST search showed that CrRVCh01 shares $27.7 \%$ identity and $48.0 \%$ similarity with the virus Lepeophtheirus salmonis rhabdovirus No127 (LSRVNo127) present in L. salmonis in the North Atlantic, while the amino acid sequence similarity to both Bivens Arm virus and Tibrogargan virus (genus Tibrovirus) is $40.0 \%$. The $\mathrm{N}$ protein contains the sequence ${ }_{283}$ QLSTRSPYST $_{292}$, which shares high similarity with the RNA-binding motif $(\mathrm{G}(\mathrm{L} / \mathrm{I})$ SXKSPYSS) present in vesiculoviruses, ephemeroviruses and lyssaviruses [19].

The putative phosphoprotein gene (P gene) of CrRV-Ch01 is $807 \mathrm{nt}$ long and contains a single ORF of $759 \mathrm{nt}$ encoding a putative protein of 253 aa (accession number: APF32074). It contains 35 potential serine/threonine phosphorylation sites and five tyrosine phosphorylation sites. This putative $P$ protein sequence shares no significant sequence identity to any sequences in the GenBank database and its identity is only suggested based on its size and genome position.

The putative matrix protein gene ( $\mathrm{M}$ gene) in CrRV-Ch01 is $739 \mathrm{nt}$ long and contains a single ORF of $687 \mathrm{nt}$ encoding a putative protein of 229 aa (accession number: APF32075). Amino acid sequence comparison with other rhabdoviruses showed that it shares $24.6 \%$ and $25.1 \%$ identity and $42.4 \%$ and $40.2 \%$ similarity with the matrix protein genes of the salmon louse rhabdoviruses LSRV-No127 (AIY25914) and Lepeophtheirus salmonis rhabdovirus No9 (LSRV-No9) (AIY25909), respectively, but no significant match with other rhabdoviruses. A potential domain, PPPY/P(S/T)AP,
Table 1 Primers and probes for TaqMan real-time RT PCR assays targeting the $\mathrm{N}$ protein gene of CrRv-Ch01,18S from $C$. rogercresseyi, and primers used for sequencing of the genome termini

\begin{tabular}{ll}
\hline Code & Sequence \\
\hline CrRV-1-NF & 5'-TGG ATT CTT TCC CCC ATT G-3' \\
CrRV-1-Nprobe & 5'-ATC GGA CGA ACT GAT-3' \\
CrRV-1-NR & 3'-TCT TGT CCT GGC TCC ATA GTG-5' \\
Crog-18sF & 5'-AGC GTC TAG CTA CAC GAG ATT GAG-3' \\
Crog-18sprobe & 5'-AAT AAC AGG TCT GTG ATG CC-3' \\
Crog-18sR & 3'-CGT GCA GCC CAG AAC ATC TA-5' \\
CRRV1_end_fw & 5'-TGGAATCTCTCGGGCAAGG-3' \\
CRRV1_end_fw_nested & 5'-TGGATTCGAGTCCTGTCTCG-3' \\
CRRV1_end_rev & 5'-CATCTCGAGTCAATACCTCC-3' \\
CRRV1_end_rev_nested & 5'-CGAAAGCTAACACATCAGGC-3' \\
\hline
\end{tabular}


which is necessary for efficient budding of the virus [20], could be represented by the sequence ${ }_{14}$ PVASAPTVPGP $_{29}$.

The putative unknown protein gene (U gene) in CrRVCh01 is 439 nt long with a single ORF of 408 nt encoding a putative protein of 136 aa (accession number: APF32076). The $\mathrm{U}$ gene nucleotide sequence and the putative protein sequence share no detectable sequence similarity with any other viruses in the public databases. However, topology analysis of the putative $U$ protein using the Phobius server predict an N-terminal signal peptide from aa 1 to 19 and an ectodomain from aa 20 to 136 , but no transmembrane region. Additional protein coding genes have been described previously in several rhabdovirus genomes, including genes inserted between the M and G genes [21-29]. They may occur as alternative or overlapping ORFs within the structural genes or as independent ORFs flanked by transcription initiation and termination sequences, as is the case with the putative U protein gene in CrRV-Ch01 [4, 5, 23, 24]. A protein with a signal peptide at this position has been described in tupaia rhabdovirus, TUPV [24]. However, unlike the $\mathrm{U}$ protein in $\mathrm{CrRV}-\mathrm{Ch} 01$ the additional protein in TUPV contain a transmembrane region. A putative protein with a signal peptide and no transmembrane region has been found in Oak Vale virus in the same genomic position as the $\mathrm{U}$ protein gene of CrRV-Ch01 [26]. While the function is unknown, the signal peptide suggests that it may be secreted from infected cells.

The glycoprotein gene (G gene) in CrRV-Ch01 is 1647 nt long and contains a single ORF of $1611 \mathrm{nt}$ encoding a putative protein of 537 amino acids (accession number: APF32077). Topology analysis using the Phobius server predicted an N-terminal signal peptide (aa 1-17), an ectodomain from aa 18 to 493, transmembrane region spanning from aa 494 to 516, and a C-terminal cytoplasmic tail from aa 517 to 537. The protein is predicted to contain five putative N-glycosylation sites, ${ }_{140} \mathrm{NDSD},{ }_{242} \mathrm{NKTS},{ }_{368} \mathrm{NKSS}$, ${ }_{420} \mathrm{NDSS}$, and ${ }_{476} \mathrm{NGTS}$, respectively. BLAST searches showed that $\mathrm{CrRV}$-Ch01 shares the highest amino acid sequence identity (22.3\%) with a virus detected in a salmon louse (L. salmonis), LSRV-No127 (accession no. KJ958536, unclassified rhabdovirus). The amino acid sequence identity to the classified anguillid perhabdovirus (accession no. AIY29111) is 20.7\%.

The L gene of CrRV-Ch01 is $6429 \mathrm{nt}$ long and contains a single ORF of $6390 \mathrm{nt}$ encoding a putative protein of 2130 aa (accession no. APF32078). The $\mathrm{L}$ protein gene shows a clear affinity to those of other members of the family Rhabdoviridae, with the closest relationships to the $\mathrm{L}$ proteins of LSRV-No9 (44.2\% identity) and LSRV-No127 (44.0\% identity). The vesiculoviruses Maraba virus, Cocal virus, Perinet virus, and Indiana virus are slightly more distant, showing $38.3,38.3,38.6$, and $38.5 \%$ identity, respectively. Six blocks of conserved sequences are shared among the
L proteins of members of the family Rhabdoviridae [30]. These blocks were also identified in the $\mathrm{CrRv}-\mathrm{Ch} 01 \mathrm{~L}$ protein after pairwise alignments with $\mathrm{L}$ proteins from related rhabdoviruses (data not shown).

To investigate the relationship of CrRV-Ch01 to other members of the family Rhabdoviridae, a phylogenetic tree was generated based on the L protein (Fig. 1). One hundred twenty-five sequences representing all of the genera of the family Rhabdoviridae were aligned using MAFFT. Ambiguously aligned regions in the alignment were removed using TrimAl [31], resulting in a sequence alignment of 1571 amino acids. Phylogenetic relationships were determined using RAxML (Randomized Axelerated Maximum Likelihood) v8.2.10 [32], available at CIPRES, employing the LG model of amino acid substitution [33]. The tree was based on approximate maximum-likelihood values using the selected model of substitution and rate heterogeneity. The robustness of each node was determined using 1000 bootstrap steps. The phylogenetic analysis showed that $\mathrm{CrRV}-\mathrm{Ch} 01$ groups in a clade with two other rhabdoviruses from a parasitic copepod (L. salmonis) present on salmon in the North Atlantic. The phylogeny of these rhabdoviruses from copepods shows no clear affinity to members of any of the known rhabdovirus genera.

To estimate the prevalence of CrRV-Ch01 in adult $C$. rogercresseyi from the three locations in Chile, 51 lice were individually tested for the presence of the virus genome sequence, using a qPCR assay targeting the $\mathrm{N}$ gene. Two real-time RT PCR assays with TaqMan probes were developed based on the putative nucleoprotein gene sequence obtained from the rhabdovirus CrRV-Ch01, present in $C$. rogercresseyi, and the $18 \mathrm{~S}$ (small subunit rRNA) from $C$. rogercresseyi (Table 1). Both assays were optimized for real-time RT PCR, and the assay targeting the $18 \mathrm{~S}$ from $C$. rogercresseyi (Crog-18S) was used as internal control. The real-time RT-PCR reaction was run in a $12.5-\mu 1$ reaction mixture containing $6.25 \mu \mathrm{l}$ of $2 \mathrm{X}$ RT-PCR, $1.0 \mu \mathrm{l}$ of $10 \mathrm{mM}$ forward primer, 1.0 of $\mu \mathrm{l} 10 \mathrm{mM}$ reverse primer, $0.22 \mu \mathrm{l}$ of $10 \mathrm{mM}$ probe, $0.25 \mu \mathrm{l}$ of $25 \mathrm{X}$ RT-PCR enzyme mix, $1.75 \mu \mathrm{l}$ of RNase-free water and $2.0 \mu \mathrm{l}$ of template. The real-time RT-PCR analysis was run using an Applied Biosystems 7500 Fast Real-Time PCR System under the following conditions: reverse transcription at $45^{\circ} \mathrm{C}$ for $10 \mathrm{~min}$, polymerase activation at $95^{\circ} \mathrm{C}$ for $10 \mathrm{~min}$, and 45 cycles of DNA dissociation at $95^{\circ} \mathrm{C}$ for 15 and annealing/elongation at $60^{\circ} \mathrm{C}$ for $45 \mathrm{~s}$. The virus was present in both male and female lice from all three locations in Chile. The prevalence of positive lice was $72 \%$ (36 of 50 lice) and ranged from 64.2 to $85.7 \%$ for the three locations. The $\mathrm{CT}$ value of positive lice ranged from 12.6 to 35.8 and the average $\mathrm{CT}$ value was 27.7 , ranging from 25.2 to 29.9 for the three locations. Four individuals tested strongly positive $(\mathrm{Ct}<15)$ for the virus. The internal control assay, Crog-18S, gave $\mathrm{Ct}$ values ranging from 4.3 to 


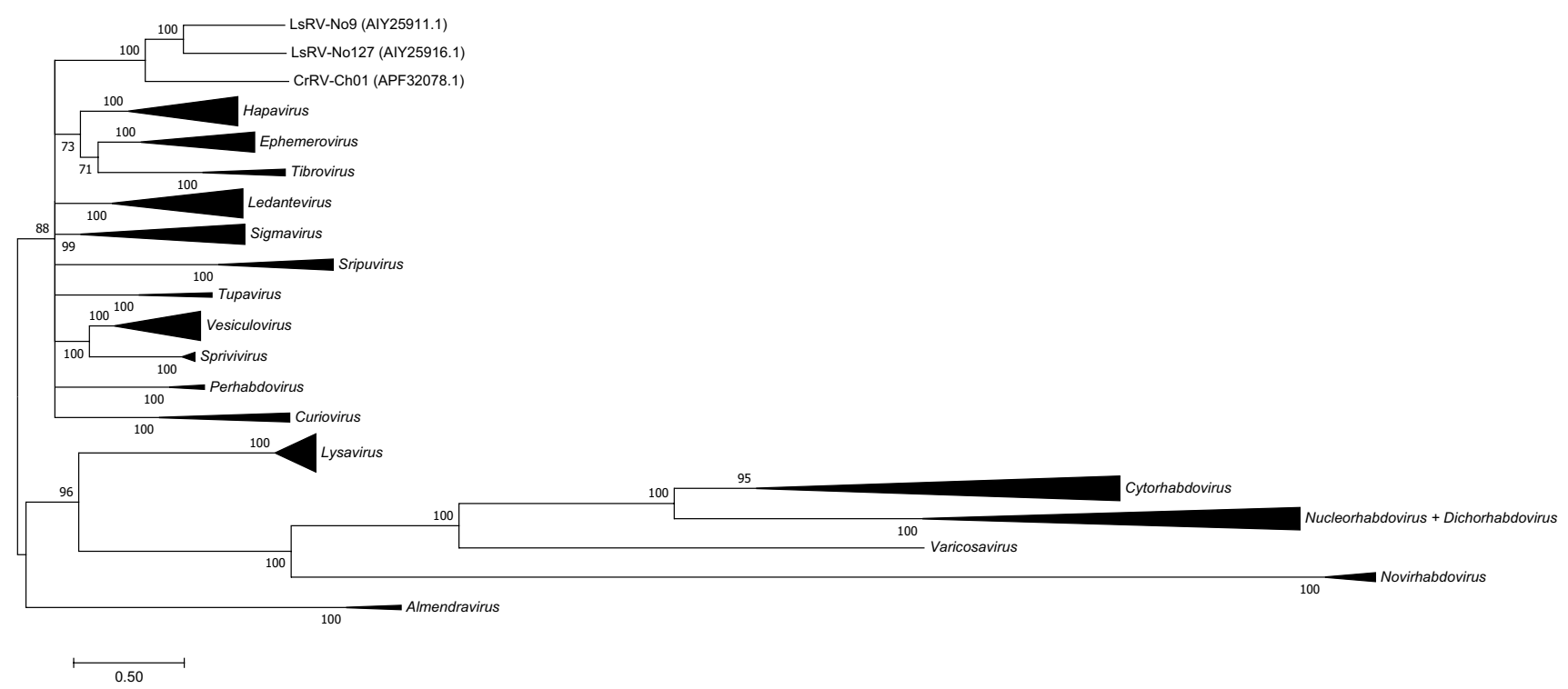

Fig. 1 Phylogenetic position of CrRV-Ch01 (accession number: APF32078.1) obtained from Caligus rogercresseyi in relation to other rhabdoviruses based on analysis of the $\mathrm{L}$ protein sequences after removal of ambiguously aligned regions using TrimAl [31]. The evolutionary relationship is presented as a maximum-likelihood tree based on 1571 aa from the complete alignment of the L protein amino acid sequences with a bootstrap support cutoff of $70 \%$. Branch

10.0 (average, 5.7) showing the presence of RNA in all samples. Viral RNA was also detected in egg strings from three positive female lice ( $\mathrm{Ct}$ values ranging from 30.6 to 31.6). The presence of viral RNA in egg strings could indicate, as described for the L. salmonis rhabdoviruses [15, 34], that vertical transmission may occur in addition to horizontal transmission.

To summarize, we have characterized the first rhabdovirus genome from the parasitic copepod Caligus rogercresseyi, CrRV-Ch01, collected from farmed Atlantic salmon in Chile. CrRV-Ch01 was not present in all the specimens of $C$. rogercresseyi tested, and we have found what appears to be a complete, functional genome. The completeness of the genome sequence, the presence of complete genome termini exhibiting inverse complementarity, TI and TTP sequences for all genes, and ORFs for all essential rhabdovirus proteins, makes it highly unlikely that this virus is integrated into its host genome. To yield the results of this study, an endogenous virus genome would have to be transcribed as a complete negative-sense viral genome with no post-transcriptional modifications. Thus, this study strongly suggests that CrRV-Ch01 is indeed an exogenous virus rather than another example of endogenous viral elements [8]. To support this, future studies should employ histology, in situ hybridization, and electron microscopy to examine the pathology and tropism of the virus and lengths represent relative phylogenetic distances according to maximum-likelihood estimates based on the LG model of amino acid substitution [33]. The scale bar shows the number of amino acid substitutions as a proportion of the branch lengths. CrRV-Ch01 constitutes a clade together with two rhabdoviruses, LsRV-No9 (accession no: AIY25911.1) and LsRV-No127 (accession no: AIY25916.1), from $L$. salmonis

presence of viral particles. The presence of CrRV-Ch01 in Atlantic salmon has not been studied. Because the lice feed on the mucus, skin and blood of their host, it is possible that we have detected a salmonid virus in the gut of the lice. However, the presence of the CrRV-Ch01 genome in the egg strings of the lice strongly suggests otherwise. Nevertheless, future studies should investigate the presence of CrRV-Ch01 in Atlantic salmon and other fish frequently infested with $C$. rogercresseyi. This could reveal if this is an arbovirus infecting both fish and lice, or solely a caligid virus. The closely related LsRV-No9 and LsRVNo127 have been detected in the skin of Atlantic salmon at the attachment site of chalimi, but there are no evidence of infection or replication [15].

The phylogenetic clustering of CrRV-Ch01 from Chile with the rhabdoviruses LsRV-No9 and LsRV-No127 from salmon lice in the North Atlantic, i.e., the demonstration of the presence of these related viruses over a large geographical area, suggest a Caligidae-specific association of this virus clade that has probably existed for a considerable time. This indicates that there may be several undiscovered rhabdoviruses infecting other members of the family Caligidae.

Acknowledgements We thank Cermaq for providing the material for this study. 


\section{Compliance with ethical standards}

Conflict of interest The authors declare that they have no conflicts of interest.

Ethical approval All applicable international, national, and institutional guidelines for the care and use of animals were followed.

Open Access This article is distributed under the terms of the Creative Commons Attribution 4.0 International License (http://creativeco mmons.org/licenses/by/4.0/), which permits unrestricted use, distribution, and reproduction in any medium, provided you give appropriate credit to the original author(s) and the source, provide a link to the Creative Commons license, and indicate if changes were made.

\section{References}

1. Hamilton-West $\mathrm{C}$ et al (2012) Epidemiological description of the sea lice (Caligus rogercresseyi) situation in southern Chile in August 2007. Prev Vet Med 104(3):341-345

2. González MP, Vargas-Chacoff L, Marín SL (2016) Stress response of Salmo salar (Linnaeus 1758) when heavily infested by Caligus rogercresseyi (Boxshall \& Bravo 2000) copepodids. Fish Physiol Biochem 42(1):263-274

3. Oelckers K et al (2014) Caligus rogercresseyi as a potential vector for transmission of Infectious Salmon Anaemia (ISA) virus in Chile. Aquaculture 420:126-132

4. Li CX et al (2015) Unprecedented genomic diversity of RNA viruses in Arthropods reveals the ancestry of negative-sense RNA viruses. eLife 4:e05378

5. Shi M et al (2016) Redefining the invertebrate RNA virosphere. Nature 540(7634):539-543

6. Katzourakis A, Gifford RJ (2010) Endogenous viral elements in animal genomes. PLoS Genet 6(11):e1001191

7. Holmes EC (2011) The evolution of endogenous viral elements. Cell Host Microbe 10(4):368-377

8. Fort $\mathrm{P}$ et al (2011) Fossil rhabdoviral sequences integrated into arthropod genomes: ontogeny, evolution and potential functionality. Mol Biol Evol 29:226

9. Ballinger MJ, Bruenn JA, Taylor DJ (2012) Phylogeny, integration and expression of sigma virus-like genes in Drosophila. Mol Phylogenet Evol 65(1):251-258

10. Feschotte C, Gilbert C (2012) Endogenous viruses: insights into viral evolution and impact on host biology. Nat Rev Genet 13(4):283-296

11. Thézé $J$ et al (2014) Remarkable diversity of endogenous viruses in a Crustacean genome. Genome Biol Evol 6(8):2129-2140

12. Aiewsakun P, Katzourakis A (2015) Endogenous viruses: connecting recent and ancient viral evolution. Virology 479-480:26-37

13. Metegnier $\mathrm{G}$ et al (2015) Comparative paleovirological analysis of crustaceans identifies multiple widespread viral groups. Mob DNA 6(1): 16

14. Geisler C, Jarvis DL (2016) Rhabdovirus-like endogenous viral elements in the genome of Spodoptera frugiperda insect cells are actively transcribed: implications for adventitious virus detection. Biologicals 44(4):219-225
15. Økland AL et al (2014) Genomic characterization and phylogenetic position of two new species in Rhabdoviridae infecting the parasitic Copepod, Salmon louse (Lepeophtheirus salmonis). PLoS One 9(11):e112517

16. Altschul SF et al (1990) Basic local alignment search tool. J Mol Biol 215(3):403-410

17. Hall TA (1999) BioEdit: a user-friendly biological sequence alignment editor and analysis program for Windows 95/98/NT. Nucleic Acids Symp Ser 41:95-98

18. Nolden $\mathrm{T}$ et al (2016) Reverse genetics in high throughput: rapid generation of complete negative strand RNA virus cDNA clones and recombinant viruses thereof. Sci Rep 6:23887

19. Walker PJ et al (1994) Structural and antigenic analysis of the nucleoprotein of bovine ephemeral fever rhabdovirus. J Gen Virol 75(Pt 8):1889-1899

20. Freed EO (2002) Viral late domains. J Virol 76(10):4679-4687

21. Quan P-L et al (2010) Moussa virus: a new member of the Rhabdoviridae family isolated from Culex decens mosquitoes in Cote d'Ivoire. Virus Res 147(1):17-24

22. Tao J-J et al (2008) Genomic sequence of mandarin fish rhabdovirus with an unusual small non-transcriptional ORF. Virus Res 132(1):86-96

23. Walker PJ et al (2015) Evolution of genome size and complexity in the Rhabdoviridae. PLoS Pathog 11(2):e1004664

24. Springfeld C, Darai G, Cattaneo R (2005) Characterization of the Tupaia rhabdovirus genome reveals a long open reading frame overlapping with $\mathrm{P}$ and a novel gene encoding a small hydrophobic protein. J Virol 79(11):6781-6790

25. Walker PJ et al (2011) Rhabdovirus accessory genes. Virus Res 162(1):110-125

26. Quan P-L et al (2011) Genetic characterization of K13965, a strain of Oak Vale virus from Western Australia. Virus Res 160(1):206-213

27. Gubala A et al (2010) Ngaingan virus, a macropod-associated rhabdovirus, contains a second glycoprotein gene and seven novel open reading frames. Virology 399(1):98-108

28. Gubala A et al (2011) Tibrogargan and coastal plains rhabdoviruses: genomic characterization, evolution of novel genes and seroprevalence in Australian livestock. J Gen Virol 92(9):2160-2170

29. Allison $\mathrm{AB}$ et al (2011) Characterization of Durham virus, a novel rhabdovirus that encodes both a $\mathrm{C}$ and $\mathrm{SH}$ protein. Virus Res 155(1):112-122

30. Poch O et al (1990) Sequence comparison of five polymerases (L proteins) of unsegmented negative-strand RNA viruses: theoretical assignment of functional domains. J Gen Virol 71(5):1153-1162

31. Capella-Gutiérrez S, Silla-Martínez JM, Gabaldón T (2009) tri$\mathrm{mAl}$ : a tool for automated alignment trimming in large-scale phylogenetic analyses. Bioinformatics 25(15):1972-1973

32. Stamatakis A (2014) RAxML version 8: a tool for phylogenetic analysis and post-analysis of large phylogenies. Bioinformatics 30(9):1312-1313

33. Le SQ, Gascuel O (2008) An improved general amino acid replacement matrix. Mol Biol Evol 25(7):1307-1320

34. Øvergård A-C et al (2017) RNAi-mediated treatment of two vertically transmitted rhabdovirus infecting the salmon louse (Lepeophtheirus Salmonis). Sci Rep 7(1):14030 